Bull. Korean Math. Soc. 52 (2015), No. 5, pp. 1401-1422

http://dx.doi.org/10.4134/BKMS.2015.52.5.1401

\title{
RESULTS ON MEROMORPHIC FUNCTIONS SHARING THREE VALUES WITH THEIR DIFFERENCE OPERATORS
}

\author{
XiaO-Min Li, Hong-Xun Yi, And Cong-Yun Kang
}

\begin{abstract}
Under the restriction of finite order, we prove two uniqueness theorems of nonconstant meromorphic functions sharing three values with their difference operators, which are counterparts of Theorem 2.1 in [6] for a finite-order meromorphic function and its shift operator.
\end{abstract}

\section{Introduction and main results}

In this paper, by meromorphic functions we will always mean meromorphic functions in the complex plane. We adopt the standard notations of the Nevanlinna theory of meromorphic functions as explained in [5], [10] and [16]. It will be convenient to let $E$ denote any set of positive real numbers of finite linear measure, not necessarily the same at each occurrence. For a nonconstant meromorphic function $h$, we denote by $T(r, h)$ the Nevanlinna characteristic of $h$ and by $S(r, h)$ any quantity satisfying $S(r, h)=o(T(r, h))$, as $r \rightarrow \infty, r \notin E$.

Let $f$ and $g$ be two nonconstant meromorphic functions, and let $a$ be a value in the extended plane. We say that $f$ and $g$ share the value $a \mathrm{CM}$, provided that $f$ and $g$ have the same $a$-points with the same multiplicities. We say that $f$ and $g$ share the value $a$ IM, provided that $f$ and $g$ have the same $a$-points ignoring multiplicities (cf. [16]). Throughout this paper, we denote by $\rho(f)$ the order of $f$ (cf. [5], [10] and [16]). We also need the following two definitions:

Definition $1.1([15])$. Let $f$ be a nonconstant meromorphic function. We define difference operators of $f$ as

$$
\Delta_{\eta} f(z)=f(z+\eta)-f(z) \text { and } \Delta_{\eta}^{n} f(z)=\Delta_{\eta}^{n-1}\left(\Delta_{\eta} f(z)\right),
$$

where $\eta$ is a nonzero complex number, $n \geq 2$ is a positive integer. If $\eta=1$, we denote $\Delta_{\eta} f(z)=\Delta f(z)$.

Received November 10, 2011; Revised January 3, 2015.

2010 Mathematics Subject Classification. 30D35, 30D30.

Key words and phrases. meromorphic functions, difference operators, uniqueness theorems.

This work is supported by the NSFC (Grant No. 11171184), the NSFC (Grant No. 11461042), the NSF of Shandong Province, China (Grant No. ZR2014AM011) and the NSF of Shandong Province, China (Grant No. ZR2013AM026). 
Remark 1.1. Definition 1.1 implies $\Delta_{\eta}^{n} f(z)=\sum_{j=0}^{n}\left(\begin{array}{c}n \\ j\end{array}\right)(-1)^{n-j} f(z+j \eta)$.

Definition $1.2([8])$. Let $k$ be a nonnegative integer or infinity. For any $a \in C \cup\{\infty\}$, we denote by $E_{k}(a, f)$ the set of all $a$-points of $f$, where an $a$ point of multiplicity $m$ is counted $m$ times if $m \leq k$, and $k+1$ times if $m>k$. If $E_{k}(a, f)=E_{k}(a, g)$, we say that $f, g$ share the value $a$ with weight $k$.

Remark 1.2. Definition 1.2 implies that if $f, g$ share a value $a$ with weight $k$, then $z_{0}$ is a zero of $f-a$ with multiplicity $m(\leq k)$ if and only if it is a zero of $g-a$ with multiplicity $m(\leq k)$, and $z_{0}$ is a zero of $f-a$ with multiplicity $m(>k)$, if and only if it is a zero of $g-a$ with multiplicity $n(>k)$, where $m$ is not necessarily equal to $n$. Throughout this paper, we write $f, g$ share $(a, k)$ to mean that $f, g$ share the value $a$ with weight $k$. Clearly, if $f, g$ share $(a, k)$, then $f, g$ share $(a, p)$ for all integer $p, 0 \leq p<k$. Also we note that $f, g$ share a value $a$ IM or CM if and only if $f, g$ share $(a, 0)$ or $(a, \infty)$, respectively.

Recently the value distribution theory of difference polynomials, Nevanlinna characteristic of $f(z+\eta)$, Nevanlinna theory for the difference operator and the difference analogue of the lemma on the logarithmic derivative has been established (cf. [2], [3], [4], [11] and [12]). Using these theories, uniqueness questions of meromorphic functions sharing values with their shifts have been recently treated as well (cf. [6], [7] and [19]). In this paper, we will consider a uniqueness question of meromorphic functions of finite orders sharing three values with their difference operators.

We recall the following result due to Heittokangas-Korhonen- Laine-Rieppo $[6]$ :

Theorem A ([6, Theorem 2.1]). Let $f$ be a meromorphic function of finite order, and let $\eta$ be a nonzero complex number. If $f(z)$ and $f(z+\eta)$ share $a_{1}$, $a_{2}, a_{3} C M$, where $a_{1}, a_{2} a_{3}$ are three distinct finite values, then $f(z)=f(z+\eta)$ for all $z \in \mathbb{C}$.

Theorem A gives a sufficient condition for a finite-order meromorphic function and its shift to be identical. One may ask: What can be said about the conclusion of Theorem A if we replace " $f(z+\eta)$ " with $\Delta_{\eta} f(z)$ ? In this direction, we will prove the following theorem, which gives a counterpart of Theorem A for finite-order meromorphic functions and their first order difference operators:

Theorem 1.1. Let $f$ be a nonconstant meromorphic function of finite order, and let $\eta$ be a nonzero complex number. If $f$ and $\Delta_{\eta} f$ share $a_{1}, a_{2}$ and $a_{3} C M$, where $a_{1}, a_{2}, a_{3}$ are three distinct values in the extended complex plane. Then $2 f(z)=f(z+\eta)$ for all $z \in \mathbb{C}$.

From Theorem 1.1 we get the following result:

Corollary 1.1. Let $f$ be a nonconstant entire function of finite order, and let $\eta$ be a nonzero complex number. If $f$ and $\Delta_{\eta} f$ share $a_{1}$ and $a_{2} C M$, where $a_{1}$ and $a_{2}$ are two distinct finite values in the complex plane. Then $2 f(z)=f(z+\eta)$ for all $z \in \mathbb{C}$. 
Proceeding as in the proof of Theorem 1.1 in Section 3 of the present paper, we can get the following more general result by Remark 2.1, Lemma 2.1, Lemmas 2.6 and 2.7 in Section 2 of the present paper:

Theorem 1.2. Let $f$ be a nonconstant meromorphic function of finite order, and let $\eta$ be a nonzero complex number. If $f$ and $\Delta_{\eta} f$ share $\left(a_{1}, k_{1}\right),\left(a_{2}, k_{2}\right)$ and $\left(a_{3}, k_{3}\right)$, where $a_{1}, a_{2}, a_{3}$ are three distinct values in the extended complex plane, and $k_{1}, k_{2}, k_{3}$ are three positive integers satisfying

$$
k_{1}+k_{2}+k_{3}>k_{1} k_{2} k_{3}+2 .
$$

Then $2 f(z)=f(z+\eta)$ for all $z \in \mathbb{C}$.

\section{Some lemmas}

In this section, we will give the following lemmas which play an important role in proving the main results of the present paper:

Lemma 2.1 ([13, Lemma 2.2]). Let $f$ and $g$ be two nonconstant rational functions that share $\left(0, k_{1}\right),\left(1, k_{2}\right)$ and $\left(\infty, k_{3}\right)$, where $k_{1}, k_{2}, k_{3}$ are three positive integers satisfying (1.1). Then $f=g$.

Lemma 2.2 ([17, Lemma 1$])$. Let $f$ and $g$ be two distinct nonconstant meromorphic functions sharing 0,1 and $\infty C M$. Then there exist two entire functions $\alpha$ and $\beta$ such that

$$
f=\frac{e^{\alpha}-1}{e^{\beta}-1}, \quad g=\frac{e^{-\alpha}-1}{e^{-\beta}-1},
$$

where $e^{\beta} \not \equiv 1, e^{\alpha} \not \equiv 1$ and $e^{\beta-\alpha} \not \equiv 1$, and $T(r, g)+T\left(r, e^{\alpha}\right)+T\left(r, e^{\beta}\right)=$ $O(T(r, f))$, as $r \notin E$ and $r \rightarrow \infty$, where $E \subset \mathbb{R}^{+}$is a subset which has a finite linear measure.

Lemma 2.3 ([1]). Let $f$ and $g$ be two distinct nonconstant meromorphic functions such that $f$ and $g$ share $0,1, \infty$ CM. If $f$ is a Möbius transformation of $g$, then $f$ and $g$ assume one of the following six relations: (i) $f g=1$; (ii) $(f-1)(g-1)=1$; (iii) $f+g=1$; (iv) $f=c g$; (v) $f-1=c(g-1)$; (vi) $[(c-1) f+1][(c-1) g-c]=-c$; where $c$ is a complex number satisfying $c \neq 0,1$.

Lemma 2.4 ([16, Theorem 1.62]). Let $f_{1}, f_{2}, \ldots, f_{n}$ be nonconstant meromorphic functions, and let $f_{n+1} \not \equiv 0$ be a meromorphic function such that $\sum_{j=1}^{n+1} f_{j}=1$. If there exists a subset $I \subseteq \mathbb{R}^{+}$satisfying mesI $=\infty$ such that

$$
\sum_{i=1}^{n+1} N\left(r, \frac{1}{f_{i}}\right)+n \sum_{i=1, i \neq j}^{n+1} \bar{N}\left(r, f_{i}\right)<(\lambda+o(1)) T\left(r, f_{j}\right), \quad j=1,2, \ldots, n,
$$

as $r \rightarrow \infty$ and $r \in I$, where $\lambda<1$. Then $f_{n+1}=1$. 
Lemma 2.5 ([2, Corollary 2.5]). Let $f$ be a nonconstant meromorphic function of finite order, and let $\eta$ be a nonzero complex number. Then for any positive number $\varepsilon$, we have

$$
m\left(r, \frac{f(z+\eta)}{f(z)}\right)+m\left(r, \frac{f(z)}{f(z+\eta)}\right)=O\left(r^{\rho(f)-1+\varepsilon}\right) .
$$

Lemma 2.6 ([9, Lemma 6$])$. Let $f$ and $g$ be two distinct nonconstant meromorphic functions such that $f$ and $g$ share $0,1, \infty$ IM. If $f$ is a quasi-Möbius transformation of $g$, then $f$ and $g$ assume one of the following six relations:
(i) $f \cdot g=1$;
(iii) $f+g=1$;
(ii) $(f-1)(g-1)=1$;
(v) $f-1=c(g-1)$,
(iv) $f=c g$;
(vi) $\quad[(c-1) f+1] \cdot[(c-1) g-c]=-c$;

where $c \not \equiv 0,1, \infty$ is a small function of $f$ and $g$.

Lemma 2.7 ([18, Lemma 2.6]). Let $f$ and $g$ be two distinct nonconstant meromorphic functions that share $\left(0, k_{1}\right),\left(1, k_{2}\right)$ and $\left(\infty, k_{3}\right)$, where $k_{1}, k_{2}$ and $k_{3}$ are three positive integers satisfying (1.1). Then

(i) $\bar{N}_{(2}\left(r, \frac{1}{f}\right)+\bar{N}_{(2}\left(r, \frac{1}{f-1}\right)+\bar{N}_{(2}(r, f)=S(r, f)$;

(ii) $\bar{N}_{(2}\left(r, \frac{1}{g}\right)+\bar{N}_{(2}\left(r, \frac{1}{g-1}\right)+\bar{N}_{(2}(r, g)=S(r, f)$.

Remark 2.1. Suppose that $f$ and $g$ in Lemma 2.7 are distinct transcendental meromorphic functions of finite order. Then, from the proof of Lemma 2.6 [18] we can find that

$$
\bar{N}_{(2}\left(r, \frac{1}{f}\right)+\bar{N}_{(2}\left(r, \frac{1}{f-1}\right)+\bar{N}_{(2}(r, f)=O(\log r)
$$

and

$$
\bar{N}_{(2}\left(r, \frac{1}{f}\right)+\bar{N}_{(2}\left(r, \frac{1}{f-1}\right)+\bar{N}_{(2}(r, f)=O(\log r)
$$

as $r \rightarrow \infty$. Therefore, in the same manner as in the proof of Lemma 1 [17] we have from (2.2) and (2.3) that

$$
f=\frac{h_{1} e^{\hat{\alpha}}-1}{h_{2} e^{\hat{\beta}}-1}, \quad g=\frac{h_{1}^{-1} e^{-\hat{\alpha}}-1}{h_{2}^{-1} e^{-\hat{\beta}}-1},
$$

where $h_{1}$ and $h_{2}$ are two non-vanishing rational functions, $\hat{\alpha}$ and $\hat{\beta}$ are nonconstant polynomials such that $h_{1} e^{\hat{\alpha}} \not \equiv 1, h_{2} e^{\hat{\beta}} \not \equiv 1$ and $e^{\hat{\beta}-\hat{\alpha}} \not \equiv \frac{h_{1}}{h_{2}}$, and $T(r, g)+T\left(r, e^{\hat{\alpha}}\right)+T\left(r, e^{\hat{\beta}}\right)=O(T(r, f))$ as $r \rightarrow \infty$.

\section{Proof of theorems}

Proof of Theorem 1.1. First of all, we set

$$
g=\Delta_{\eta} f .
$$

Suppose that $f$ and $g$ are rational functions. Then, by Lemma 2.1 and the assumptions of Theorem 1.1 we have $f=g$. Combining this with (3.1), we get 
the conclusion of Theorem 1.1. Next we suppose that $f$ and $g$ are transcendental meromorphic functions such that $f \not \equiv g$. We consider the following two cases.

Case 1. Suppose that $g$ is a Möbius transformation of $f$. We discuss the following two subcases.

Subcase 1.1. Suppose that one of $a_{1}, a_{2}$ and $a_{3}$ is $\infty$, say $a_{3}=\infty$. Without loss of generality, we let $a_{1}=0, a_{2}=1$ and $a_{3}=\infty$. From Lemma 2.2 we have (2.1). By Lemma 2.3 we know that $f, g$ satisfy one of the six relations (i)-(vi) of Lemma 2.3.

Suppose that $f, g$ satisfy one of the relations (i), (ii) and (vi) of Lemma 2.3. Then $\infty$ is a Picard exceptional value of $f$ and $g$, and so $f$ and $g$ are entire functions. In fact, if $f$ and $g$ satisfy the relation (i) of Lemma 2.3, then

$$
f(z)=e^{\gamma_{1}(z)}, \quad f(z+\eta)-f(z)=e^{-\gamma_{1}(z)}
$$

for all $z \in \mathbb{C}$, where $\gamma_{1}(z)$ is a nonconstant polynomial. By (3.2) we deduce $e^{\gamma_{1}(z)+\gamma_{1}(z+\eta)}-e^{2 \gamma_{1}(z)}=1$ for all $z \in \mathbb{C}$, this together with Lemma 2.4 implies a contradiction. If $f$ and $g$ satisfy (ii) of Lemma 2.3, then

$$
f(z)=1+e^{\gamma_{2}(z)}, \quad f(z+\eta)-f(z)=1+e^{-\gamma_{2}(z)}
$$

for all $z \in \mathbb{C}$, where $\gamma_{2}(z)$ is a nonconstant polynomial. From (3.3) we deduce

$$
e^{\gamma_{2}(z+\eta)}-e^{\gamma_{2}(z)}-e^{-\gamma_{2}(z)}=1
$$

for all $z \in \mathbb{C}$, which together with Lemma 2.4 yields a contradiction. Similarly, if $f$ and $g$ satisfy (vi) of Lemma 2.3, we also get a contradiction.

Suppose that $f$ and $g$ satisfy (iii) of Lemma 2.3. Then 0, 1 are Picard exceptional values of $f$ and $g$. Hence $f=(f-1) e^{\gamma_{3}}$, where $\gamma_{3}$ is a nonconstant polynomial. Combining this with (3.1) and (iii) of Lemma 2.3, we have $e^{\gamma_{3}(z+\eta)} /\left(e^{\gamma_{3}(z+\eta)}-1\right)=1$ for all $z \in \mathbb{C}$, which is impossible.

Suppose that $f, g$ satisfy (iv) of Lemma 2.3. Then 1 and $c$ are Picard exceptional values of $f$. Hence $f-1=(f-c) e^{\gamma_{4}}$, where $\gamma_{4}$ is a nonconstant polynomial. Hence $f=\left(1-c e^{\gamma_{4}}\right) /\left(1-e^{\gamma_{4}}\right)$, this together with (3.1) and (iv) of Lemma 2.3 gives

$$
c^{2} e^{\gamma_{4}(z)}+\left(1+c-c^{2}\right) e^{\gamma_{4}(z+\eta)}-c e^{\gamma_{4}(z)+\gamma_{4}(z+\eta)}=1
$$

for all $z \in \mathbb{C}$. From (3.4) and Lemma 2.4 we can get a contradiction.

Suppose that $f, g$ satisfy (v) of Lemma 2.3. By substituting (2.1) into (v) of Lemma 2.3 we can get $e^{\alpha}=c$, and so it follows from (2.1), (3.1) and (v) of Lemma 2.3 that

$$
c e^{\beta(z+\eta)-\beta(z)}-e^{\beta(z+\eta)}=c-1
$$

for all $z \in \mathbb{C}$. The only possibly constant term of the left side of $(3.5)$ is $c e^{\beta(z+\eta)-\beta(z)}$. This together with Lemma 2.4 and $c \neq 1$ gives $e^{\beta(z+\eta)-\beta(z)}=$ $c-1$, and so $e^{\beta(z+\eta)}=0$ for all $z \in \mathbb{C}$, which is impossible. 
Subcase 1.2. Suppose that none of $a_{1}, a_{2}$ and $a_{3}$ is $\infty$. We set

$$
H(z)=\frac{f(z)-a_{1}}{f(z)-a_{3}} \cdot \frac{a_{2}-a_{3}}{a_{2}-a_{1}}, \quad K(z)=\frac{\Delta_{\eta} f(z)-a_{1}}{\Delta_{\eta} f(z)-a_{3}} \cdot \frac{a_{2}-a_{3}}{a_{2}-a_{1}} .
$$

From (3.6) and the condition that $f$ and $\Delta_{\eta} f$ share $a_{1}, a_{2}, a_{3}$ CM we know that $H$ and $K$ share $0,1, \infty$ CM. From (3.1) and $f \not \equiv g$ we deduce $H \not \equiv K$. Hence we get from Lemma 2.2 that

$$
H=\frac{e^{\alpha_{1}}-1}{e^{\beta_{1}}-1}, \quad K=\frac{e^{-\alpha_{1}}-1}{e^{-\beta_{1}}-1},
$$

where $\alpha_{1}$ and $\beta_{1}$ are polynomials such that $e^{\beta_{1}} \not \equiv 1, e^{\alpha_{1}} \not \equiv 1, e^{\beta_{1}-\alpha_{1}} \not \equiv 1$ and $T(r, K)+T\left(r, e^{\alpha_{1}}\right)+T\left(r, e^{\beta_{1}}\right)=O(T(r, f))$ as $r \rightarrow \infty$. By the condition that $\Delta_{\eta} f$ is a Möbius transformation of $f$ we know that $K$ is a Möbius transformation of $H$. By Lemma 2.3 we consider the following six subcases.

Subcase 1.2.1. Suppose that $H$ and $K$ satisfy $H K=1$. Then

$$
H(z)=e^{\gamma_{5}(z)} \text { and } K(z)=e^{-\gamma_{5}(z)},
$$

where $\gamma_{5}$ is a nonconstant polynomial. From the left equalities of (3.6) and (3.8) we get

$$
f(z)=\frac{a_{3} a_{4} e^{\gamma_{5}(z)}-a_{1}}{a_{4} e^{\gamma_{5}(z)}-1}
$$

for all $z \in \mathbb{C}$, where

$$
a_{4}=\frac{a_{2}-a_{1}}{a_{2}-a_{3}}
$$

From (3.9) we get

$$
f(z+\eta)-f(z)=\frac{a_{4}\left(a_{1}-a_{3}\right)\left(e^{\gamma_{5}(z+\eta)}-e^{\gamma_{5}(z)}\right)}{\left(a_{4} e^{\gamma_{5}(z)}-1\right)\left(a_{4} e^{\gamma_{5}(z+\eta)}-1\right)}
$$

for all $z \in \mathbb{C}$. Meanwhile, from (3.11) and the right equalities of (3.6) and (3.8) we get

$$
\frac{f(z+\eta)-f(z)-a_{1}}{f(z+\eta)-f(z)-a_{3}}=a_{4} e^{-\gamma_{5}(z)}
$$

for all $z \in \mathbb{C}$. By substituting (3.11) into (3.12) we get

$$
\begin{aligned}
& \left(a_{3} a_{4}-2 a_{1} a_{4}-a_{3} a_{4}^{3}\right) e^{\gamma_{5}(z)+\gamma_{5}(z+\eta)}-a_{3} a_{4} e^{2 \gamma_{5}(z)}+a_{1} a_{4}^{2} e^{2 \gamma_{5}(z)+\gamma_{5}(z+\eta)} \\
& +a_{1} a_{4}^{2} e^{\gamma_{5}(z+\eta)}+\left(2 a_{3} a_{4}^{2}-a_{1} a_{4}^{2}\right) e^{\gamma_{5}(z)}=a_{3} a_{4}
\end{aligned}
$$

for all $z \in \mathbb{C}$. By rewriting (3.13) we get

$$
b_{1}(z) e^{3 \gamma_{5}(z)}+b_{2}(z) e^{2 \gamma_{5}(z)}+b_{3}(z) e^{\gamma_{5}(z)}=a_{3} a_{4}
$$

for all $z \in \mathbb{C}$, where

$$
b_{1}(z)=a_{1} a_{4}^{2} e^{\gamma_{5}(z+\eta)-\gamma_{5}(z)},
$$




$$
b_{2}(z)=\left(a_{3} a_{4}-2 a_{1} a_{4}-a_{3} a_{4}^{3}\right) e^{\gamma_{5}(z+\eta)-\gamma_{5}(z)}-a_{3} a_{4}
$$

and

$$
b_{3}(z)=a_{1} a_{4}^{2} e^{\gamma_{5}(z+\eta)-\gamma_{5}(z)}+2 a_{3} a_{4}^{2}-a_{1} a_{4}^{2}
$$

for all $z \in \mathbb{C}$. From (3.15)-(3.17) and Lemma 2.5 we get

$$
T\left(r, b_{j}(z)\right)=O\left(r^{\operatorname{deg}\left(\gamma_{5}\right)-1+\varepsilon}\right), 1 \leq j \leq 3
$$

as $r \rightarrow \infty$, where $\varepsilon$ is an arbitrary positive number. Suppose that $b_{1} \not \equiv 0$. Applying Valiron-Mokhonko identity (cf. [14]) to (3.14) and (3.18), we get

$$
\begin{aligned}
T\left(r, b_{1}(z) e^{3 \gamma_{5}(z)}\right) & =3 T\left(r, e^{\gamma_{5}(z)}\right)+O\left(r^{\operatorname{deg}\left(\gamma_{5}\right)-1+\varepsilon}\right) \\
& =T\left(r, a_{3} a_{4}-b_{3}(z) e^{\gamma_{5}(z)}-b_{2}(z) e^{2 \gamma_{5}(z)}\right) \\
& \leq 2 T\left(r, e^{\gamma_{5}(z)}\right)+O\left(r^{\operatorname{deg}\left(\gamma_{5}\right)-1+\varepsilon}\right),
\end{aligned}
$$

which implies that

$$
T\left(r, e^{\gamma_{5}(z)}\right)=O\left(r^{\operatorname{deg}\left(\gamma_{5}\right)-1+\varepsilon}\right) .
$$

But this means that $\gamma_{5}(z)$ is a constant, which is impossible. Hence $b_{1}=0$. Similarly $b_{2}=b_{3}=0$. Combining this with (3.14) and (3.15), we get $a_{1} a_{4}^{2}=$ $a_{3} a_{4}=0$. From (3.10) we get $a_{4} \neq 0,1$. Hence $a_{1}=a_{3}=0$, which is impossible.

Subcase 1.2.2. Suppose that $H$ and $K$ satisfy $(H-1)(K-1)=1$. Then $\infty$ and 1 are Picard exceptional values. Hence

$$
H(z)=1+e^{\gamma_{6}(z)}, \quad K(z)=1+e^{-\gamma_{6}(z)},
$$

where $\gamma_{6}$ is a nonconstant polynomial. From (3.10) and the left equalities of (3.6) and (3.19) we get

$$
f(z)=\frac{\left(a_{1}-a_{3} a_{4}\right)-a_{3} a_{4} e^{\gamma_{6}(z)}}{\left(1-a_{4}\right)-a_{4} e^{\gamma_{6}(z)}} .
$$

From (3.20) we get

$$
f(z+\eta)-f(z)=\frac{a_{4}\left(a_{1}-a_{3}\right)\left[e^{\gamma_{6}(z+\eta)}-e^{\gamma_{6}(z)}\right]}{\left(1-a_{4}\right)^{2}+a_{4}\left(a_{4}-1\right)\left[e^{\gamma_{6}(z)}+e^{\gamma_{6}(z+\eta)}\right]+a_{4}^{2} e^{\gamma_{6}(z)+\gamma_{6}(z+\eta)}} .
$$

From (3.10), Definition 1.2 and the right equalities of (3.6) and (3.19) we get

$$
f(z+\eta)-f(z)=\frac{\left(a_{1}-a_{3} a_{4}\right)-a_{3} a_{4} e^{-\gamma_{6}(z)}}{\left(1-a_{4}\right)-a_{4} e^{-\gamma_{6}(z)}} .
$$

From (3.21) and (3.22) we get

(3.23) $c_{1} e^{2 \gamma_{6}(z)+\gamma_{6}(z+\eta)}+c_{2} e^{\gamma_{6}(z)+\gamma_{6}(z+\eta)}+c_{3} e^{2 \gamma_{6}(z)}+c_{4} e^{\gamma_{6}(z+\eta)}+c_{5} e^{\gamma_{6}(z)}=c_{6}$ for all $z \in \mathbb{C}$, where

$$
\begin{gathered}
c_{1}=c_{4}=\left(a_{3} a_{4}-a_{1}\right) a_{4}^{2}, \\
c_{2}=2 a_{1} a_{4}-a_{3} a_{4}-2 a_{1} a_{4}^{2}+2 a_{3} a_{4}^{3},
\end{gathered}
$$




$$
\begin{gathered}
c_{3}=a_{3} a_{4}\left(a_{4}-1\right)^{2}, \\
c_{5}=\left(a_{1}+a_{3} a_{4}-2 a_{3}\right) a_{4}^{2}
\end{gathered}
$$

and

$$
c_{6}=-a_{3} a_{4}\left(a_{4}-1\right)^{2} .
$$

We note that $\gamma_{6}(z)$ and $\gamma_{6}(z+\eta)$ are two nonconstant polynomials that have the same highest terms. Hence the non-vanishing terms on the left side of (3.23) must be nonconstant terms. This together with Lemma 2.4 and $a_{4} \neq 0,1$ gives $c_{6}=0$, and so $a_{3}=0$. Hence $(3.23)$ can be rewritten as

$(3.29) a_{4} e^{2 \gamma_{6}(z)+\gamma_{6}(z+\eta)}+2\left(a_{4}-1\right) e^{\gamma_{6}(z)+\gamma_{6}(z+\eta)}+a_{4} e^{\gamma_{6}(z+\eta)}-a_{4} e^{\gamma_{6}(z)}=0$

for all $z \in \mathbb{C}$. Next in the same manner as in Subcase 1.2.1 we get from (3.29) that $a_{4}=0$ and $a_{4}=1$, which is impossible.

Subcase 1.2.3. Suppose that $H$ and $K$ satisfy $H+K=1$. Then 0,1 are Picard exceptional values of $H$ and $K$. Hence

$$
\frac{H}{H-1}=e^{\gamma_{7}},
$$

where $\gamma_{7}$ is a nonconstant polynomial. Substituting (3.6), (3.10) into $H+K=$ 1 , we get

$$
\frac{f(z+\eta)-f(z)-a_{1}}{f(z+\eta)-f(z)-a_{3}}+\frac{f(z)-a_{1}}{f(z)-a_{3}}=a_{4} .
$$

From (3.10), (3.30) and the left equality of (3.6) we get

$$
f(z)=\frac{a_{1}+\left(a_{3} a_{4}-a_{1}\right) e^{\gamma_{7}(z)}}{1+\left(a_{4}-1\right) e^{\gamma_{7}(z)}}
$$

for all $z \in \mathbb{C}$. From (3.32) we get

$$
\frac{f(z)-a_{1}}{f(z)-a_{3}}=\frac{\left[\left(a_{3} a_{4}-1\right)-a_{1}\left(a_{4}-1\right)\right] e^{\gamma_{7}(z)}}{\left(a_{1}-a_{3}\right)+\left(a_{3}-1\right) e^{\gamma_{7}(z)}}
$$

and

$$
f(z+\eta)-f(z)=\frac{\left[a_{1}\left(a_{4}-1\right)-\left(a_{3} a_{4}-1\right)\right]\left[e^{\gamma_{7}(z)}-e^{\gamma_{7}(z+\eta)}\right]}{1+\left(a_{4}-1\right)\left[e^{\gamma_{7}(z)}+e^{\gamma_{7}(z+\eta)}\right]+\left(a_{4}-1\right)^{2} e^{\gamma_{7}(z)+\gamma_{7}(z+\eta)}}
$$

for all $z \in \mathbb{C}$. By substituting (3.33), (3.34) into (3.31) we get $(3.35)$

$$
d_{1} e^{2 \gamma_{7}(z)+\gamma_{7}(z+\eta)}+d_{2} e^{\gamma_{7}(z)+\gamma_{7}(z+\eta)}+d_{3} e^{2 \gamma_{7}(z)}+d_{4} e^{\gamma_{7}(z+\eta)}+d_{5} e^{\gamma_{7}(z)}=d_{6}
$$

for all $z \in \mathbb{C}$, where

$$
d_{1}=\left(a_{4}-1\right)^{2}\left(a_{1}+a_{3}+a_{1} a_{3} a_{4}-2 a_{1} a_{3}-a_{3} a_{4}\right),
$$


(3.37)

$$
\begin{aligned}
& d_{2}=\left(a_{1}+a_{3}-a_{1} a_{4}-1\right)\left(a_{1}+a_{4}-a_{1} a_{4}-1\right)+\left(a_{3}-a_{1}\right)\left(a_{1}-a_{3} a_{4}\right)\left(a_{4}-1\right)^{2} \\
& \quad+\left(a_{3}-1\right)\left(a_{3} a_{4}-2 a_{1} a_{4}+2 a_{1}-1\right), \\
& \begin{array}{l}
(3.38) \\
d_{3}=
\end{array} \quad\left(1-a_{3} a_{4}\right)\left(a_{3}-1\right)+\left(a_{3} a_{4}-a_{1} a_{4}+a_{1}-1\right)\left(a_{1} a_{4}+a_{3}-a_{1}-2 a_{3} a_{4}+1\right) \\
& \quad-a_{4}\left(a_{3}-1\right)\left(a_{1} a_{4}+a_{3}-a_{1}-2 a_{3} a_{4}+1\right), \\
& (3.39) \quad d_{4}=\left(a_{1}-a_{3}\right)\left(a_{1} a_{4}^{2}-3 a_{1} a_{4}+2 a_{1}+a_{4}-1\right), \\
& \text { (3.40) } \quad d_{5}=\left(a_{3}-1\right)\left(a_{3} a_{4}-a_{1}\right)+\left(a_{3}-a_{1}\right)\left(a_{4}-1\right)\left(a_{1} a_{4}-2 a_{3} a_{4}+1\right)
\end{aligned}
$$

and

$$
d_{6}=\left(a_{1}-a_{3}\right)\left(a_{1}-a_{3} a_{4}\right) .
$$

Proceeding as in Subcase 1.2.1, we get from (3.35)-(3.41) and Lemma 2.4 that

$$
d_{1}=d_{6}=0 .
$$

By (3.41) and $d_{6}=0$ we have

$$
a_{3} a_{4}=a_{1} .
$$

From (3.10) and (3.43) we get $a_{2}=0$, and so $a_{1} \neq 0$ and $a_{3} \neq 0$. Noting that $a_{4} \neq 1$, we get from (3.36), (3.43) and $d_{1}=0$ that $a_{1}^{2}=\left(2 a_{1}-1\right) a_{3}$. This together with $a_{1} \neq 0$ and $a_{3} \neq 0$ reveals that

$$
2 a_{1} \neq 1
$$

and

$$
a_{3}=\frac{a_{1}^{2}}{2 a_{1}-1}
$$

By (3.38), (3.43) and (3.45) we get

$$
d_{3}=\frac{\left(a_{1}-1\right)^{3}}{2 a_{1}-1} .
$$

From (3.45) and $a_{1} \neq a_{3}$ we have $a_{1} \neq 1$. This together (3.44) and (3.46) implies that $d_{3} \neq 0$. By (3.43) and (3.45) we get

$$
a_{4}=\frac{2 a_{1}-1}{a_{1}} \text {. }
$$

By substituting (3.45) and (3.47) into (3.37) and (3.39) we deduce $d_{2}=d_{4}=0$. Combining this with (3.42), we know that (3.35) can be rewritten as

$$
d_{3} e^{2 \gamma_{7}(z)}+d_{5} e^{\gamma_{7}(z)}=0
$$

for all $z \in \mathbb{C}$, which together with $d_{3} \neq 0$ and the standard Valiron-Mokhon'ko lemma we deduce $T\left(r, e^{\gamma_{7}(z)}\right)=S\left(r, e^{\gamma_{7}(z)}\right)$, which is impossible.

Subcase 1.2.4. Suppose that $H$ and $K$ satisfy $H=c K$. Then $1, c$ are Picard exceptional values of $H$ and $K$. Hence $H(z)-1=(H(z)-c) e^{\gamma_{8}(z)}$ for 
all $z \in \mathbb{C}$, where $\gamma_{8}(z)$ is a nonconstant polynomial. Combining $H=c K$ with (3.6), we have

$$
\frac{f(z)-a_{1}}{f\left(z-a_{3}\right)}=\frac{c\left(f(z+\eta)-f(z)-a_{1}\right)}{f(z+\eta)-f(z)-a_{3}}
$$

for all $z \in \mathbb{C}$. By substituting the left part of (3.6) into $H(z)-1=(H(z)-$ c) $e^{\gamma_{8}(z)}$ we have

$$
f(z)=\frac{a_{1}-a_{3} a_{4}+\left(c a_{3} a_{4}-a_{1}\right) e^{\gamma_{8}(z)}}{1-a_{4}+\left(c a_{4}-1\right) e^{\gamma_{8}(z)}} .
$$

From (3.49) we have

$$
f(z+\eta)-f(z)=\frac{(c-1)\left(a_{3}-a_{1}\right) a_{4}\left(e^{\gamma_{8}(z+\eta)}-e^{\gamma_{8}(z)}\right)}{\left(1-a_{4}+\left(c a_{4}-1\right) e^{\gamma_{8}(z+\eta)}\right)\left(1-a_{4}+\left(c a_{4}-1\right) e^{\gamma_{8}(z)}\right)} .
$$

By substituting (3.49) and (3.50) into (3.48), we have (3.51)

$$
h_{1} e^{\gamma_{8}(z+\eta)+2 \gamma_{8}(z)}+h_{2} e^{\gamma_{8}(z+\eta)+\gamma_{8}(z)}+h_{3} e^{2 \gamma_{8}(z)}+h_{4} e^{\gamma_{8}(z+\eta)}+h_{5} e^{\gamma_{8}(z)}=h_{6}
$$

for all $z \in \mathbb{C}$, where

$$
\begin{gathered}
h_{1}=c\left(a_{1}-a_{3} a_{4}\right)\left(c a_{4}-1\right)^{2}, \\
h_{2}=c(c-1) a_{4}\left(a_{3}-a_{1}\right)\left(a_{4}-1\right)+c\left(1-a_{4}\right)\left(c a_{4}-1\right)\left(a_{1}-a_{3} a_{4}\right) \\
-\left(c a_{4}-1\right)^{2}\left(c a_{1}-a_{3} a_{4}\right),
\end{gathered}
$$

$h_{5}=\left(1-a_{4}\right)\left(c a_{4}-1\right)\left(a_{3} a_{4}-c a_{1}\right)+c\left(a_{1}-a_{3} a_{4}\right)\left(1-a_{4}\right)^{2}+(c-1) a_{4}\left(a_{4}-c\right)\left(a_{3}-a_{1}\right)$ and

$$
h_{6}=\left(a_{3} a_{4}-c a_{1}\right)\left(1-a_{4}\right)^{2} .
$$

Proceeding as in Subcase 1.1, we get from (3.51)-(3.57) and Lemma 2.4 that $h_{1}=h_{6}=0$. This together with (3.52), (3.57) and $a_{4} \neq 1$ gives $a_{3} a_{4}=c a_{1}$, and so it follows from (3.52) that

$$
h_{1}=c(1-c) a_{1}\left(c a_{4}-1\right)^{2}=0 .
$$

Combining this with $c \neq 0$ and $c \neq 1$, we have $a_{1}=0$ or $c a_{4}=1$.

Suppose that $a_{1}=0$. Then, by $a_{3} a_{4}=c a_{1}$, and $a_{4} \neq 0$ we have $a_{3}=0$, which contradicts the assumption that $a_{1}$ and $a_{3}$ are two distinct finite values 
in the complex plane. Suppose that $c a_{4}=1$. Then, by (3.51)-(3.57) we find that (3.51) can be rewritten as

(3.58)

$$
\begin{aligned}
& c(c-1) a_{4}\left(a_{3}-a_{1}\right)\left(a_{4}-1\right) e^{\gamma_{8}(z+\eta)+\gamma_{8}(z)} \\
& +c(c-1) a_{4}\left(a_{1}-a_{3}\right)\left(a_{4}-1\right) e^{2 \gamma_{8}(z)}+(c-1) a_{4}\left(c-a_{4}\right)\left(a_{3}-a_{1}\right) e^{\gamma_{8}(z+\eta)} \\
& +\left\{\left(c a_{1}-a_{3}\right)\left(1-a_{4}\right)^{2}+(c-1) a_{4}\left(a_{4}-c\right)\left(a_{3}-a_{1}\right)\right\} e^{\gamma_{8}(z)}=0
\end{aligned}
$$

for all $z \in \mathbb{C}$. Next, in the same manner as in Subcase 1.1, we can deduce from (3.58) and Lemma 2.4 that $e^{\gamma_{8}(z+\eta)-\gamma_{8}(z)}=1$, and so (3.58) can be rewritten as

$$
\left(c a_{1}-a_{3}\right)\left(1-a_{4}\right)^{2} e^{\gamma_{8}(z)}=0
$$

for all $z \in \mathbb{C}$. Noting that $a_{4} \neq 1,0$ and $c \neq 0,1$, we have from (3.59) that $c a_{1}=a_{3}$. This together with $a_{3} a_{4}=c a_{1}$ gives $a_{1}=a_{3}=0$, which is impossible.

Subcase 1.2.5. Suppose that $H$ and $K$ satisfy

$$
H-1=c(K-1) \text {. }
$$

By substituting (3.7) into (3.60) we get $e^{\alpha_{1}}=c$, and so

$$
H=\frac{c-1}{e^{\beta_{1}}-1}, \quad K=\frac{(1-c) e^{\beta_{1}}}{c\left(1-e^{\beta_{1}}\right)}
$$

for all $z \in \mathbb{C}$. From (3.10) and the left equalities of (3.6) and (3.61) we get

$$
f(z)=\frac{a_{3} a_{4}(c-1)+a_{1}-a_{1} e^{\beta_{1}(z)}}{a_{4}(c-1)+1-e^{\beta_{1}(z)}}
$$

for all $z \in \mathbb{C}$. From (3.62) we get

$$
f(z+\eta)-f(z)=\frac{(c-1) a_{4}\left(a_{1}-a_{3}\right)\left[e^{\beta_{1}(z)}-e^{\beta_{1}(z+\eta)}\right]}{\left[1+(c-1) a_{4}-e^{\beta_{1}(z+\eta)}\right]\left[1+(c-1) a_{4}-e^{\beta_{1}(z)}\right]}
$$

for all $z \in \mathbb{C}$. From the condition $a_{1} \neq a_{3}$ and the right equalities of (3.6) and (3.61) we get

$$
f(z+\eta)-f(z)=\frac{\left[(c-1) a_{3} a_{4}-c a_{1}\right] e^{\beta_{1}(z)}+c a_{1}}{\left.\left[(c-1) a_{4}-c\right)\right] e^{\beta_{1}(z)}+c}
$$

for all $z \in \mathbb{C}$. From (3.63) and (3.64) we get

$(3.65) j_{1} e^{2 \beta_{1}(z)+\beta_{1}(z+\eta)}+j_{2} e^{\beta_{1}(z)+\beta_{1}(z+\eta)}+j_{3} e^{2 \beta_{1}(z)}+j_{4} e^{\beta_{1}(z)}+j_{5} e^{\beta_{1}(z+\eta)}=j_{6}$ for all $z \in \mathbb{C}$, where

$$
j_{1}=c a_{1}-(c-1) a_{3} a_{4},
$$

$j_{2}=\left\{(c-1) a_{3} a_{4}-c a_{1}\right\}\left\{1+(c-1) a_{4}\right\}-(c-1) a_{4}\left(a_{1}-a_{3}\right)\left\{(c-1) a_{4}-c\right\}-c a_{1}$,

$(3.68) j_{3}=(c-1) a_{4}\left(a_{1}-a_{3}\right)\left\{(c-1) a_{4}-c\right\}+\left\{(c-1) a_{3} a_{4}-c a_{1}\right\}\left\{1+(c-1) a_{4}\right\}$,

$$
j_{4}=c(c-1) a_{4}\left(a_{1}-a_{3}\right),
$$




$$
j_{5}=c a_{1}\left\{1+(c-1) a_{4}\right\}-c(c-1) a_{4}\left(a_{1}-a_{3}\right)
$$

and

$$
j_{6}=c a_{1}\left\{1+(c-1) a_{4}\right\}^{2} .
$$

Proceeding as in Subcase 1.2.1 we get from (3.51)-(3.57) and Lemma 2.4 that $j_{1}=j_{6}=0$, and so $1+(c-1) a_{1}=c a_{1}-(c-1) a_{3} a_{4}=0$. Hence $(3.65)$ can be rewritten as

$$
\begin{aligned}
& \left\{\left(a_{1}-a_{3}\right)\left[(c-1) a_{4}-c\right]+a_{3}\right\} e^{\beta_{1}(z)+\beta_{1}(z+\eta)}+\left(a_{3}-a_{1}\right)\left[(c-1) a_{4}-c\right] e^{2 \beta_{1}(z)} \\
& +c\left(a_{3}-a_{1}\right) e^{\beta_{1}(z)}+c\left(a_{1}-a_{3}\right) e^{\beta_{1}(z+\eta)}=0
\end{aligned}
$$

for all $z \in \mathbb{C}$. Proceeding as in Subcase 1.2.1, we get from (3.70) that

$$
e^{\beta_{1}(z+\eta)-\beta_{1}(z)}=\frac{\left(a_{1}-a_{3}\right)\left[(c-1) a_{4}-c\right]}{\left(a_{1}-a_{3}\right)\left[(c-1) a_{4}-c\right]+a_{3}}=1,
$$

which implies that $a_{3}=0$. Combining this with $c a_{1}-(c-1) a_{3} a_{4}=0$ and $c \neq 0$, we get $a_{1}=a_{3}=0$, which is impossible.

Subcase 1.2.6. Suppose that $H$ and $K$ satisfy

$$
[(c-1) H+1][(c-1) K-c]=-c .
$$

By substituting (3.7) into (3.73) we get

$$
c e^{\alpha_{1}-\beta_{1}}-e^{\alpha_{1}}-e^{\beta_{1}-\alpha_{1}}+e^{\beta_{1}}+c e^{-\alpha_{1}}-c e^{-\beta_{1}}=c-1 .
$$

By (3.6), (3.7) and the standard Valiron-Mokhon'ko lemma we know that at least one of $e^{\alpha_{1}}$ and $e^{\beta_{1}}$ is not a constant.

Suppose that $e^{\beta_{1}}$ is not a constant, $e^{\alpha_{1}}$ is a constant. Then from (3.74), Lemma 2.4 and the supposition $H \not \equiv K$ we get $e^{\alpha_{1}}=-c$. Hence we get from (3.7) that

$$
H-1=-c(K-1) \text {. }
$$

Proceeding as in Subcase 1.2.3, we get a contradiction from (3.75).

Suppose that $e^{\beta_{1}}$ is a constant, $e^{\alpha_{1}}$ is not a constant. Then from (3.74), Lemma 2.4 and the supposition $H \not \equiv K$ we get $e^{\beta_{1}}=-1$ or $e^{\beta_{1}}=c$, from which we will derive a contradiction. In fact, if $e^{\beta_{1}}=-1$, from (3.10) and the left equalities of (3.6) and (3.7) we get

$$
f(z+\eta)-f(z)=\frac{2 a_{4}\left(a_{1}-a_{3}\right)\left[e^{\alpha_{1}(z)}-e^{\alpha_{1}(z+\eta)}\right]}{\left(2-a_{4}\right)^{2}+a_{4}\left(2-a_{4}\right)\left[e^{\alpha_{1}(z)}+e^{\alpha_{1}(z+\eta)}\right]+a_{4}^{2} e^{\alpha_{1}(z)+\alpha_{1}(z+\eta)}} .
$$

From (3.10) and the right equalities of (3.6) and (3.7) we get from $e^{\beta_{1}}=-1$ that

$$
f(z+\eta)-f(z)=\frac{\left(a_{3} a_{4}-2 a_{1}\right)-a_{3} a_{4} e^{-\alpha_{1}(z)}}{\left(a_{4}-2\right)-a_{4} e^{-\alpha_{1}(z)}} .
$$


From (3.76) and (3.77) we get

(3.78)

$k_{1} e^{2 \alpha_{1}(z)+\alpha_{1}(z+\eta)}+k_{2} e^{\alpha_{1}(z)+\alpha_{1}(z+\eta)}+k_{3} e^{2 \alpha_{1}(z)}+k_{4} e^{\alpha_{1}(z+\eta)}+k_{5} e^{\alpha_{1}(z)}=k_{6}$,

where

$$
\begin{gathered}
k_{1}=\left(a_{3} a_{4}-2 a_{1}\right) a_{4}^{2}, \\
k_{2}=\left(2-a_{4}\right)\left(a_{3} a_{4}-2 a_{1}\right) a_{4}+2\left(a_{1}-a_{3}\right)\left(a_{4}-2\right) a_{4}-a_{3} a_{4}^{3}, \\
k_{3}=\left(2-a_{4}\right)\left(a_{3} a_{4}-2 a_{1}\right) a_{4}-2\left(a_{1}-a_{3}\right)\left(a_{4}-2\right) a_{4}, \\
k_{4}=2\left(a_{3}-a_{1}\right) a_{4}^{2}+a_{3} a_{4}^{2}\left(a_{4}-2\right), \\
k_{5}=a_{3} a_{4}^{2}\left(a_{4}-2\right)+\left(a_{3} a_{4}-2 a_{1}\right)\left(2-a_{4}\right)^{2}+2\left(a_{1}-a_{3}\right) a_{4}^{2}
\end{gathered}
$$

and

$$
k_{6}=a_{3} a_{4}\left(a_{4}-2\right)^{2} .
$$

Proceeding as in Subcase 1.2.1 we get from (3.76)-(3.84) and Lemma 2.4 that $k_{1}=k_{6}=0$. Hence

$$
a_{3} a_{4}-2 a_{1}=a_{3} a_{4}\left(a_{4}-2\right)^{2}=0 .
$$

Noting that $a_{1} \neq a_{3}$ and $a_{4} \neq 0,1$, we get from (3.85) that $a_{4}=2$, and so $a_{1}=a_{3}$, which is impossible. Similarly we can get a contradiction if $e^{\beta_{1}}=c$.

Suppose that $e^{\alpha_{1}}$ and $e^{\beta_{1}}$ are not constants. Then from (3.74), Lemma 2.4 and the supposition $H \not \equiv K$ we get $c e^{\alpha_{1}-\beta_{1}}=-1$, this together with (3.7) gives $c H=-K$. Next in the same manner as in Subcase 1.2.4 we can get a contradiction.

Case 2. Suppose that $g$ is not a Möbius transformation of $f$. We consider the following two subcases.

Subcase 2.1. Suppose that one of $a_{1}, a_{2}$ and $a_{3}$ is $\infty$, say $a_{3}=\infty$. Without loss of generality, we let $a_{1}=0, a_{2}=1$ and $a_{3}=\infty$. Then we have (2.1). From (2.1) and the above supposition we deduce that none of $e^{\alpha}, e^{\beta}, e^{\beta-\alpha}$ is a constant. From (2.1), (3.1) and the condition $\rho(f)<\infty$ we deduce $\rho\left(e^{\alpha}\right)<\infty$ and $\rho\left(e^{\beta}\right)<\infty$, and so $\alpha, \beta$ are polynomials. By substituting (2.1) into (3.1) we get

$$
\begin{aligned}
& (3.86) \\
& e^{\beta(z)}-e^{\beta(z+\eta)-\alpha(z+\eta)+\alpha(z)}-e^{\beta(z+\eta)-\alpha(z+\eta)+\beta(z)}+e^{\beta(z)-\alpha(z)+\beta(z+\eta)-\alpha(z+\eta)} \\
& +e^{\beta(z+\eta)-\alpha(z+\eta)}+e^{\alpha(z)-\alpha(z+\eta)}-e^{\beta(z)-\alpha(z)-\alpha(z+\eta)} \equiv 1 .
\end{aligned}
$$

Then the only possibly constant terms in the left sides of (3.86) are $-e^{\beta(z+\eta)-\alpha(z+\eta)+\alpha(z)},-e^{\beta(z+\eta)-\alpha(z+\eta)+\beta(z)}, e^{\alpha(z)-\alpha(z+\eta)},-e^{\beta(z)-\alpha(z)-\alpha(z+\eta)}$.

We discuss the following four subcases.

Subcase 2.1.1. Suppose that $e^{\beta(z+\eta)-\alpha(z+\eta)+\alpha(z)}$ is a constant. Then

$$
\operatorname{deg}(\beta) \leq \operatorname{deg}(\alpha)-1 .
$$


From (3.86), (3.87) and the above supposition we deduce that the only possibly constant term on the left side of $(3.86)$ is $-e^{\beta(z+\eta)-\alpha(z+\eta)+\alpha(z)}$, this together with Lemma 2.4 gives

$$
e^{\beta(z+\eta)-\alpha(z+\eta)+\alpha(z)} \equiv-1
$$

and

$$
\begin{aligned}
& e^{\beta(z+\eta)-\alpha(z+\eta)}-e^{\beta(z+\eta)-\alpha(z+\eta)-\alpha(z)}-e^{\beta(z+\eta)-\beta(z)-\alpha(z+\eta)} \\
& -e^{\alpha(z)-\alpha(z+\eta)-\beta(z)}+e^{-\alpha(z)-\alpha(z+\eta)} \equiv 1
\end{aligned}
$$

From (3.87), (3.89) and the above supposition we deduce that the only possibly constant term on the left side of $(3.8)$ is $-e^{\alpha(z)-\alpha(z+\eta)-\beta(z)}$. Hence we get from Lemma 2.4 that $e^{\alpha(z)-\alpha(z+\eta)-\beta(z)} \equiv-1$. Combining this with (3.88), we get $e^{\beta(z)+\beta(z+\eta)} \equiv 1$, which is impossible.

Subcase 2.1.2. Suppose that $e^{\beta(z+\eta)-\alpha(z+\eta)+\alpha(z)}$ is not a constant and that $e^{\beta(z+\eta)-\alpha(z+\eta)+\beta(z)}$ is a constant. Then the highest term of $\alpha$ is equal to 2 times of the highest term of $\beta$. Hence $\operatorname{deg}(\alpha)=\operatorname{deg}(\beta)$ and the orders of other terms of the left side of (3.86) apart from $e^{\beta(z+\eta)-\alpha(z+\eta)+\beta(z)}$ and $e^{\alpha(z)-\alpha(z+\eta)}$ are equal to $\operatorname{deg}(\alpha)$, while the order of the term $e^{\alpha(z)-\alpha(z+\eta)}$ is smaller than $\operatorname{deg}(\alpha)$. This together with Lemma 2.4 gives

$$
e^{\alpha(z)-\alpha(z+\eta)}-e^{\beta(z+\eta)-\alpha(z+\eta)+\beta(z)} \equiv 1
$$

and

$$
\begin{aligned}
& e^{\beta(z+\eta)-\alpha(z+\eta)+\alpha(z)-\beta(z)}-e^{\beta(z+\eta)-\alpha(z)-\alpha(z+\eta)}-e^{\beta(z+\eta)-\beta(z)-\alpha(z+\eta)} \\
& +e^{-\alpha(z)-\alpha(z+\eta)} \equiv 1
\end{aligned}
$$

From the above analysis we know that the only possibly constant term of the left side of $(3.91)$ is $e^{\beta(z+\eta)-\alpha(z+\eta)+\alpha(z)-\beta(z)}$. This together with Lemma 2.4 gives

$$
e^{\beta(z+\eta)-\alpha(z+\eta)+\alpha(z)-\beta(z)} \equiv 1, \quad e^{-\beta(z+\eta)}-e^{\alpha(z)-\beta(z)} \equiv 1 .
$$

From Lemma 2.4 and the right equality of (3.92) we get $e^{-\beta(z+\eta)} \equiv 0$, which is impossible.

Subcase 2.1.3. Suppose that $e^{\alpha(z)-\alpha(z+\eta)}$ is a constant, while

$$
e^{\beta(z+\eta)-\alpha(z+\eta)+\alpha(z)} \text { and } e^{\beta(z+\eta)-\alpha(z+\eta)+\beta(z)}
$$

are not constants.

Subcase 2.1.3.1. Suppose that $e^{\beta(z)-\alpha(z)-\alpha(z+\eta)}$ is a constant. Then the highest term of $\beta$ is equal to 2 times the highest term of $\alpha$. This together with (3.86) and Lemma 2.4 gives

$$
e^{\alpha(z)-\alpha(z+\eta)}-e^{\beta(z)-\alpha(z)-\alpha(z+\eta)} \equiv 1
$$

and

$$
e^{\alpha(z+\eta)-\beta(z+\eta)}-e^{\alpha(z)-\beta(z)}+e^{-\alpha(z)}+e^{-\beta(z)} \equiv 1 .
$$


From (3.94), Lemma 2.4 and the above supposition we get a contradiction.

Subcase 2.1.3.2. Suppose that $e^{\beta(z)-\alpha(z)-\alpha(z+\eta)}$ is not a constant. Then from (3.86), Lemma 2.4 and the above supposition we get

$$
e^{\alpha(z)-\alpha(z+\eta)} \equiv 1
$$

and

$$
\begin{aligned}
& e^{\beta(z+\eta)-\alpha(z+\eta)+\alpha(z)-\beta(z)}+e^{\beta(z+\eta)-\alpha(z+\eta)}-e^{-\alpha(z)+\beta(z+\eta)-\alpha(z+\eta)} \\
& -e^{\beta(z+\eta)-\alpha(z+\eta)-\beta(z)}+e^{-\alpha(z)-\alpha(z+\eta)} \equiv 1 .
\end{aligned}
$$

By substituting (3.95) into (3.96) we get

$$
e^{2 \alpha(z)}+e^{\beta(z+\eta)}+e^{\beta(z+\eta)-\beta(z)+\alpha(z)}-e^{\beta(z+\eta)+\alpha(z)}-e^{2 \alpha(z)+\beta(z+\eta)-\beta(z)} \equiv 1 .
$$

Noting that none of $e^{\alpha}, e^{\beta}, e^{\beta-\alpha}$ is a constant, we deduce from (3.97) that at most one of $e^{\beta(z+\eta)-\beta(z)+\alpha(z)}, e^{\beta(z+\eta)+\alpha(z)}, e^{2 \alpha(z)+\beta(z+\eta)-\beta(z)}$, say

$$
e^{2 \alpha(z)+\beta(z+\eta)-\beta(z)} \text { is a constant. }
$$

This together with Lemma 2.4 gives

$$
e^{2 \alpha(z)+\beta(z+\eta)-\beta(z)} \equiv-1
$$

and

$$
e^{2 \alpha(z)}+e^{\beta(z+\eta)}+e^{\beta(z+\eta)-\beta(z)+\alpha(z)}-e^{\beta(z+\eta)+\alpha(z)}=0 .
$$

Multiplying two sides of (3.99) by $e^{-\beta(z+\eta)-\alpha(z)}$ and noting (3.95), we get

$$
e^{\alpha(z+\eta)-\beta(z+\eta)}+e^{-\alpha(z)}+e^{-\beta(z)} \equiv 1 .
$$

From (3.100), Lemma 2.4 and the above supposition we get a contradiction.

Subcase 2.1.4. Suppose that $e^{\beta(z)-\alpha(z)-\alpha(z+\eta)}$ is a constant, while $e^{\alpha(z)-\alpha(z+\eta)}, e^{\beta(z+\eta)-\alpha(z+\eta)+\alpha(z)}, e^{\beta(z+\eta)-\alpha(z+\eta)+\beta(z)}$ are not constants.

Then it follows from (3.86), Lemma 2.4 and the above supposition that

$$
e^{\beta(z)-\alpha(z)-\alpha(z+\eta)} \equiv-1
$$

and

$$
\begin{aligned}
& \text { (3.102) } \\
& \begin{array}{l}
e^{\beta(z)}-e^{\beta(z+\eta)-\alpha(z+\eta)+\alpha(z)}-e^{\beta(z+\eta)-\alpha(z+\eta)+\beta(z)}+e^{\beta(z)-\alpha(z)+\beta(z+\eta)-\alpha(z+\eta)} \\
+e^{\beta(z+\eta)-\alpha(z+\eta)}+e^{\alpha(z)-\alpha(z+\eta)} \equiv 0 .
\end{array}
\end{aligned}
$$

By substituting (3.101) into (3.102) we get

(3.103)

$$
\begin{aligned}
& e^{2 \alpha(z+\eta)+\alpha(z+2 \eta)}+e^{2 \alpha(z+\eta)}-e^{\alpha(z+\eta)+\alpha(z+2 \eta)}-e^{2 \alpha(z+\eta)+\alpha(z+2 \eta)-\alpha(z)} \\
& +e^{\alpha(z+\eta)+\alpha(z+2 \eta)-\alpha(z)} \equiv 1 .
\end{aligned}
$$

By rewriting (3.103) we have

$$
l_{1}(z) e^{3 \alpha(z)}+l_{2}(z) e^{2 \alpha(z)}+l_{3}(z) e^{\alpha(z)}=1,
$$


where

$$
l_{1}(z)=e^{2 \alpha(z+\eta)+\alpha(z+2 \eta)-3 \alpha(z)},
$$

$(3.106) l_{2}(z)=e^{2 \alpha(z+\eta)-2 \alpha(z)}-e^{\alpha(z+\eta)+\alpha(z+2 \eta)-2 \alpha(z)}-e^{2 \alpha(z+\eta)+\alpha(z+2 \eta)-3 \alpha(z)}$

and

$$
l_{3}(z)=e^{\alpha(z+\eta)+\alpha(z+2 \eta)-2 \alpha(z)} .
$$

From (3.105)-(3.107) and Lemma 2.5 we get

$$
T\left(r, l_{j}(z)\right)=O\left(r^{\operatorname{deg}(\alpha)-1+\varepsilon}\right), 1 \leq j \leq 3
$$

as $r \rightarrow \infty$, where $\varepsilon$ is an arbitrary positive number. Next in the same manner as in Subcase 1.2.1 we can get from (3.104)-(3.108) that $l_{1}=l_{2}=l_{3}=0$, which contradicts (3.105) and (3.107).

Subcase 2.2. Suppose that none of $a_{1}, a_{2}$ and $a_{3}$ is $\infty$. We set (3.6). In the same manner as in Subcase 1.2 we have (3.7), where $\alpha_{1}$ and $\beta_{1}$ are polynomials such that none of $e^{\beta_{1}}, e^{\alpha_{1}}, e^{\beta_{1}-\alpha_{1}}$ is a constant, and such that $T(r, K)+T\left(r, e^{\alpha_{1}}\right)+T\left(r, e^{\beta_{1}}\right)=O(T(r, f))$ as $r \rightarrow \infty$. From the left equalities of (3.6) and (3.7) we get

$$
\left(1-\frac{a_{4} e^{\alpha_{1}(z)}-a_{4}}{e^{\beta_{1}(z)}-1}\right) f(z)=a_{1}-\frac{a_{3} a_{4} e^{\alpha_{1}(z)}-a_{3} a_{4}}{e^{\beta_{1}(z)}-1} .
$$

From (3.109) and $a_{1} \neq a_{3}$ we have

$$
f(z)=\frac{a_{1} e^{\beta_{1}(z)}-a_{3} a_{4} e^{\alpha_{1}(z)}+a_{3} a_{4}-a_{1}}{e^{\beta_{1}(z)}-a_{4} e^{\alpha_{1}(z)}+a_{4}-1}
$$

and

$$
f(z+\eta)=\frac{a_{1} e^{\beta_{1}(z+\eta)}-a_{3} a_{4} e^{\alpha_{1}(z+\eta)}+a_{3} a_{4}-a_{1}}{e^{\beta_{1}(z+\eta)}-a_{4} e^{\alpha_{1}(z+\eta)}+a_{4}-1}
$$

for all $z \in \mathbb{C}$, where $a_{4}$ is defined as in (3.10). From (3.7), (3.10) and the right equalities of (3.6) we get

$$
\frac{f(z+\eta)-f(z)-a_{1}}{f(z+\eta)-f(z)-a_{3}}=\frac{a_{4} e^{-\alpha_{1}(z)}-a_{4}}{e^{-\beta_{1}(z)}-1} .
$$

By substituting (3.110) and (3.111) into (3.112) we get

$$
\begin{aligned}
& a_{1} a_{4}^{2} e^{\alpha_{1}(z+\eta)-\beta_{1}(z+\eta)}+\left(a_{1} a_{4}^{2}+a_{3} a_{4}\right) e^{-\beta_{1}(z)}+\left(a_{3} a_{4}-a_{1} a_{4}^{2}\right) e^{-\beta_{1}(z+\eta)} \\
& +2 a_{4}\left(a_{1} a_{4}-2 a_{1}+a_{3}\right) e^{\alpha_{1}(z)-\beta_{1}(z)+\alpha_{1}(z+\eta)-\beta_{1}(z+\eta)}-a_{3} a_{4} e^{2 \alpha_{1}(z)-2 \beta_{1}(z)} \\
& +\left(a_{3} a_{4}-a_{1}\right) e^{\alpha_{1}(z)-2 \beta_{1}(z)} \\
& +\left(2 a_{1} a_{4}-a_{1} a_{4}^{2}-a_{3} a_{4}\right) e^{\alpha_{1}(z)+\alpha_{1}(z+\eta)-2 \beta_{1}(z)-\beta_{1}(z+\eta)} \\
& +\left(a_{3} a_{4}-a_{1} a_{4}^{2}\right) e^{2 \alpha_{1}(z)-2 \beta_{1}(z)-\beta_{1}(z+\eta)} \\
& +\left(2 a_{1} a_{4}-a_{3} a_{4}-a_{1} a_{4}^{2}\right) e^{\alpha_{1}(z)+\alpha_{1}(z+\eta)-\beta_{1}(z+\eta)}
\end{aligned}
$$




$$
\begin{aligned}
& +\left(a_{1} a_{4}^{2}-2 a_{3} a_{4}^{2}+a_{3} a_{4}\right) e^{2 \alpha_{1}(z)-\beta_{1}(z)}-a_{3} a_{4}\left(a_{4}-1\right)^{2} e^{2 \alpha_{1}(z)-\beta_{1}(z)-\beta_{1}(z+\eta)} \\
& +a_{1}\left(a_{4}-1\right)^{2} e^{\alpha_{1}(z)-\beta_{1}(z+\eta)}+a_{4}\left(a_{1}-a_{3}\right)\left(a_{4}+1\right) e^{\alpha_{1}(z)-\beta_{1}(z)-\beta_{1}(z+\eta)} \\
& -a_{3} a_{4}\left(a_{4}-1\right)^{2} e^{-\beta_{1}(z)-\beta_{1}(z+\eta)}+a_{1} a_{4}^{2} e^{2 \alpha_{1}(z)+\alpha_{1}(z+\eta)-2 \beta_{1}(z)-\beta_{1}(z+\eta)} \\
& +a_{1}\left(a_{4}-1\right)^{2} e^{\alpha_{1}(z)-2 \beta_{1}(z)-\beta_{1}(z+\eta)}+\left(a_{3} a_{4}-a_{1}\right) e^{\alpha_{1}(z)} \\
& +\left(a_{3} a_{4}-a_{1}\right) a_{4}^{2} e^{2 \alpha_{1}(z)+\alpha_{1}(z+\eta)-\beta_{1}(z+\eta)-\beta_{1}(z)} \\
& +\left(a_{3} a_{4}-a_{1}\right) a_{4}^{2} e^{\alpha_{1}(z+\eta)-\beta_{1}(z)-\beta_{1}(z+\eta)} \\
& -2\left(a_{3} a_{4}+a_{1} a_{4}^{2}-2 a_{3} a_{4}^{2}-a_{1}\right) e^{\alpha_{1}(z)-\beta_{1}(z)}=a_{3} a_{4}
\end{aligned}
$$

for all $z \in \mathbb{C}$. We discuss the following four subcases.

Subcase 2.2.1. Suppose that one of

$$
\begin{gathered}
e^{\alpha_{1}(z)-2 \beta_{1}(z)}, e^{2 \alpha_{1}(z)-\beta_{1}(z)}, e^{\alpha_{1}(z)+\alpha_{1}(z+\eta)-\beta_{1}(z+\eta)}, e^{\alpha_{1}(z)-\beta_{1}(z+\eta)}, \\
e^{\alpha_{1}(z)-\beta_{1}(z)-\beta_{1}(z+\eta)}, e^{\alpha_{1}(z)-2 \beta_{1}(z)-\beta_{1}(z+\eta)}, e^{\alpha_{1}(z+\eta)-\beta_{1}(z)-\beta_{1}(z+\eta)}
\end{gathered}
$$

is a nonzero constant, say $e^{\alpha_{1}-2 \beta_{1}}=A_{1}$. By substituting $e^{\alpha_{1}}=A_{1} e^{2 \beta_{1}}$ into (3.113) we have

$$
\begin{aligned}
& s_{1} e^{4 \beta_{1}(z)+2 \beta_{1}(z+\eta)}+s_{2} e^{4 \beta_{1}(z)+\beta_{1}(z+\eta)}+s_{3} e^{3 \beta_{1}(z)+2 \beta_{1}(z+\eta)}+s_{4} e^{3 \beta_{1}(z)+\beta_{1}(z+\eta)} \\
& +s_{5} e^{2 \beta_{1}(z)+2 \beta_{1}(z+\eta)}+s_{6} e^{4 \beta_{1}(z)}+s_{7} e^{3 \beta_{1}(z)}+s_{8} e^{2 \beta_{1}(z)+\beta_{1}(z+\eta)} \\
& +s_{9} e^{\beta_{1}(z)+2 \beta_{1}(z+\eta)}+s_{10} e^{2 \beta_{1}(z)}+s_{11} e^{\beta_{1}(z)+\beta_{1}(z+\eta)}+s_{12} e^{2 \beta_{1}(z+\eta)}+s_{13} e^{\beta_{1}(z)} \\
& +s_{14} e^{\beta_{1}(z+\eta)}=s_{15}
\end{aligned}
$$

for all $z \in \mathbb{C}$, where

$$
s_{1}=\left(a_{3} a_{4}-a_{1}\right) a_{4}^{2} A_{1}^{3}, \quad s_{2}=\left(a_{1} a_{4}^{2}-2 a_{3} a_{4}^{2}+a_{3} a_{4}\right) A_{1}^{2},
$$

(3.116) $s_{3}=A_{1}^{2}\left(2 a_{1} a_{4}-a_{3} a_{4}\right)+\left(A_{1}^{3}-A_{1}^{2}\right) a_{1} a_{4}^{2}, \quad s_{4}=\left(a_{3} a_{4}-a_{1}\right) A_{1}-a_{3} a_{4} A_{1}^{2}$,

$$
s_{5}=2 a_{4}\left(a_{1} a_{4}-2 a_{1}+a_{3}\right) A_{1}^{2}, \quad s_{6}=-a_{3} a_{4}\left(a_{4}-1\right)^{2} A_{1}^{2},
$$

$$
s_{7}=a_{3} a_{4}-a_{1} a_{4}^{2}+a_{1}\left(a_{4}-1\right)^{2} A_{1}, \quad s_{8}=-2\left(a_{3} a_{4}+a_{1} a_{4}^{2}-2 a_{3} a_{4}^{2}-a_{1}\right) A_{1},
$$

(3.119) $s_{9}=a_{1} a_{4}^{2} A_{1}+\left(2 a_{1} a_{4}-a_{1} a_{4}^{2}-a_{3} a_{4}\right) A_{1}^{2}, \quad s_{10}=a_{4}\left(a_{1}-a_{3}\right)\left(a_{4}+1\right) A_{1}$,

$$
s_{11}=\left(a_{3} a_{4}-a_{1}\right) A_{1}-a_{3} a_{4}, \quad s_{12}=\left(a_{3} a_{4}-a_{1}\right) a_{4}^{2} A_{1},
$$

$s_{13}=\left(a_{3} a_{4}-a_{1} a_{4}^{2}\right)+a_{1}\left(a_{4}-1\right)^{2} A_{1}, \quad s_{14}=a_{1} a_{4}^{2}+a_{3} a_{4}, \quad s_{15}=a_{3} a_{4}\left(a_{4}-1\right)^{2}$.

By rewriting (3.114) we get

$$
\begin{aligned}
& s_{2} e^{-\beta_{1}(z+\eta)}+s_{3} e^{-\beta_{1}(z)}+s_{4} e^{-\beta_{1}(z)-\beta_{1}(z+\eta)}+s_{5} e^{-2 \beta_{1}(z)}+s_{6} e^{-2 \beta_{1}(z+\eta)} \\
& +s_{7} e^{-\beta_{1}(z)-2 \beta_{1}(z+\eta)}+s_{8} e^{-2 \beta_{1}(z)-\beta_{1}(z+\eta)}+s_{9} e^{-3 \beta_{1}(z)}+s_{10} e^{-2 \beta_{1}(z)-2 \beta_{1}(z+\eta)}
\end{aligned}
$$




$$
\begin{aligned}
& +s_{11} e^{-3 \beta_{1}(z)-\beta_{1}(z+\eta)}+s_{12} e^{-4 \beta_{1}(z)}+s_{13} e^{-3 \beta_{1}(z)-2 \beta_{1}(z+\eta)} \\
& +s_{14} e^{-4 \beta_{1}(z)-\beta_{1}(z+\eta)}-s_{15} e^{-4 \beta_{1}(z)-2 \beta_{1}(z+\eta)}=-s_{1}
\end{aligned}
$$

for all $z \in \mathbb{C}$. From (3.114), (3.122) and in the same manner as in Subcase 1.2.1 we get $s_{1}=s_{15}=0$. This together with (3.115), (3.121) and $a_{4} \neq 0,1$ gives $a_{1}=a_{3}=0$, which is impossible.

Subcase 2.2.2. Suppose that one of

$e^{2 \alpha_{1}(z)-2 \beta_{1}(z)-\beta_{1}(z+\eta)}$ and $e^{2 \alpha_{1}(z)-\beta_{1}(z)-\beta_{1}(z+\eta)}$, say $e^{2 \alpha_{1}(z)-2 \beta_{1}(z)-\beta_{1}(z+\eta)}$ is a nonzero constant. Then

$$
e^{\alpha_{1}(z)}=A_{2} e^{2 \gamma(z)+\gamma(z+\eta)}
$$

for all $z \in \mathbb{C}$, where $A_{2} \neq 0$ is a constant. By substituting (3.123) into (3.113) we get

$$
\begin{aligned}
& \left(a_{3} a_{4}-a_{1}\right) a_{4}^{2} A_{2}^{3} e^{4 \gamma(z)+4 \gamma(z+\eta)+\gamma(z+2 \eta)}+\left(a_{1} a_{4}^{2}-2 a_{3} a_{4}^{2}+a_{3} a_{4}\right) A_{2}^{2} e^{4 \gamma(z)+4 \gamma(z+\eta)} \\
& +\left(2 a_{1} a_{4}-a_{3} a_{4}-a_{1} a_{4}^{2}\right) A_{2}^{2} e^{4 \gamma(z)+3 \gamma(z+\eta)+\gamma(z+2 \eta)}+\left(a_{3} a_{4}-a_{1}\right) A_{2} e^{4 \gamma(z)+3 \gamma(z+\eta)} \\
& +a_{1} a_{4}^{2} A_{2}^{3} e^{2 \gamma(z)+4 \gamma(z+\eta)+\gamma(z+2 \eta)}+2 a_{4}\left(a_{1} a_{4}-2 a_{1}+a_{3}\right) A_{2}^{2} e^{2 \gamma(z)+3 \gamma(z+\eta)+\gamma(z+2 \eta)} \\
& -a_{3} a_{4} A_{2}^{2} e^{2 \gamma(z)+4 \gamma(z+\eta)}-a_{3} a_{4}\left(a_{4}-1\right)^{2} A_{2}^{2} e^{4 \gamma(z)+2 \gamma(z+\eta)} \\
& +a_{1}\left(a_{4}-1\right)^{2} A_{2} e^{4 \gamma(z)+\gamma(z+\eta)}-2\left(a_{3} a_{4}+a_{1} a_{4}^{2}-2 a_{3} a_{4}^{2}-a_{1}\right) A_{2} e^{2 \gamma(z)+3 \gamma(z+\eta)} \\
& +a_{1} a_{4}^{2} A_{2} e^{2 \gamma(z)+2 \gamma(z+\eta)+\gamma(z+2 \eta)}+\left(a_{3} a_{4}-a_{1} a_{4}^{2}\right) A_{2}^{2} e^{2 \gamma(z)+2 \gamma(z+\eta)} \\
& -a_{3} a_{4} e^{2 \gamma(z)+2 \gamma(z+\eta)}+\left(2 a_{1} a_{4}-a_{1} a_{4}^{2}-a_{3} a_{4}\right) A_{2}^{2} e^{3 \gamma(z+\eta)+\gamma(z+2 \eta)} \\
& +a_{4}\left(a_{1}-a_{3}\right)\left(a_{4}+1\right) A_{2} e^{2 \gamma(z)+\gamma(z+\eta)}+\left(a_{3} a_{4}-a_{1}\right) A_{2} e^{3 \gamma(z+\eta)} \\
& +\left(a_{3} a_{4}-a_{1}\right) a_{4}^{2} A_{2} e^{2 \gamma(z+\eta)+\gamma(z+2 \eta)}+\left(a_{1} a_{4}^{2}+a_{3} a_{4}\right) e^{2 \gamma(z+\eta)} \\
& +\left(a_{3} a_{4}-a_{1} a_{4}^{2}\right) e^{2 \gamma(z)}+a_{1}\left(a_{4}-1\right)^{2} A_{2} e^{\gamma(z+\eta)}=a_{3} a_{4}\left(a_{4}-1\right)^{2}
\end{aligned}
$$

for all $z \in \mathbb{C}$. In the same manner as in Subcase 2.2.1 we get

$$
\left(a_{3} a_{4}-a_{1}\right) a_{4}^{2} A_{2}^{3}=a_{3} a_{4}\left(a_{4}-1\right)^{2}=0,
$$

which together with $a_{4} \neq 0,1$ implies that $a_{1}=a_{3}=0$, this is impossible.

Subcase 2.2.3. Suppose that

$$
\begin{aligned}
& e^{\alpha_{1}(z)-2 \beta_{1}(z)}, e^{2 \alpha_{1}(z)-\beta_{1}(z)}, e^{\alpha_{1}(z)+\alpha_{1}(z+\eta)-\beta_{1}(z+\eta)}, e^{\alpha_{1}(z)-\beta_{1}(z)-\beta_{1}(z+\eta)}, \\
& e^{\alpha_{1}(z)-\beta_{1}(z+\eta)}, e^{\alpha_{1}(z)-2 \beta_{1}(z)-\beta_{1}(z+\eta)}, e^{\alpha_{1}(z+\eta)-\beta_{1}(z)-\beta_{1}(z+\eta)}, \\
& e^{2 \alpha_{1}(z)-2 \beta_{1}(z)-\beta_{1}(z+\eta)}, e^{2 \alpha_{1}(z)-\beta_{1}(z)-\beta_{1}(z+\eta)}
\end{aligned}
$$

are not constants, and that one of

$$
e^{\alpha_{1}(z)+\alpha_{1}(z+\eta)-2 \beta_{1}(z)-\beta_{1}(z+\eta)}, e^{2 \alpha_{1}(z)+\alpha_{1}(z+\eta)-\beta_{1}(z)-\beta_{1}(z+\eta)}
$$

is a constant. 
If $e^{\alpha_{1}(z)+\alpha_{1}(z+\eta)-2 \beta_{1}(z)-\beta_{1}(z+\eta)}$ and $e^{2 \alpha_{1}(z)+\alpha_{1}(z+\eta)-\beta_{1}(z)-\beta_{1}(z+\eta)}$ are constants, then $e^{\alpha_{1}(z)+\beta_{1}(z)}$ is a constant. Next in the same manner as in the proof of Subcase 2.2.1 we get a contradiction.

If $e^{2 \alpha_{1}(z)+\alpha_{1}(z+\eta)-\beta_{1}(z)-\beta_{1}(z+\eta)}$ is not a constant, $e^{\alpha_{1}(z)+\alpha_{1}(z+\eta)-2 \beta_{1}(z)-\beta_{1}(z+\eta)}$ is a constant, then we get from (3.113) and Lemma 2.4 that

$$
\left(2 a_{1} a_{4}-a_{1} a_{4}^{2}-a_{3} a_{4}\right) e^{\alpha_{1}(z)+\alpha_{1}(z+\eta)-2 \beta_{1}(z)-\beta_{1}(z+\eta)}=a_{3} a_{4}
$$

and

$$
\begin{aligned}
& a_{1} a_{4}^{2} e^{\alpha_{1}(z+\eta)-\beta_{1}(z+\eta)}+\left(a_{1} a_{4}^{2}+a_{3} a_{4}\right) e^{-\beta_{1}(z)}+\left(a_{3} a_{4}-a_{1} a_{4}^{2}\right) e^{-\beta_{1}(z+\eta)} \\
& +2 a_{4}\left(a_{1} a_{4}-2 a_{1}+a_{3}\right) e^{\alpha_{1}(z)-\beta_{1}(z)+\alpha_{1}(z+\eta)-\beta_{1}(z+\eta)}-a_{3} a_{4} e^{2 \alpha_{1}(z)-2 \beta_{1}(z)} \\
& +\left(a_{3} a_{4}-a_{1}\right) e^{\alpha_{1}(z)-2 \beta_{1}(z)}+\left(a_{3} a_{4}-a_{1} a_{4}^{2}\right) e^{2 \alpha_{1}(z)-2 \beta_{1}(z)-\beta_{1}(z+\eta)} \\
& +\left(2 a_{1} a_{4}-a_{3} a_{4}-a_{1} a_{4}^{2}\right) e^{\alpha_{1}(z)+\alpha_{1}(z+\eta)-\beta_{1}(z+\eta)} \\
& +\left(a_{1} a_{4}^{2}-2 a_{3} a_{4}^{2}+a_{3} a_{4}\right) e^{2 \alpha_{1}(z)-\beta_{1}(z)}-a_{3} a_{4}\left(a_{4}-1\right)^{2} e^{2 \alpha_{1}(z)-\beta_{1}(z)-\beta_{1}(z+\eta)} \\
& +a_{1}\left(a_{4}-1\right)^{2} e^{\alpha_{1}(z)-\beta_{1}(z+\eta)}+a_{4}\left(a_{1}-a_{3}\right)\left(a_{4}+1\right) e^{\alpha_{1}(z)-\beta_{1}(z)-\beta_{1}(z+\eta)} \\
& -a_{3} a_{4}\left(a_{4}-1\right)^{2} e^{-\beta_{1}(z)-\beta_{1}(z+\eta)}+a_{1} a_{4}^{2} e^{2 \alpha_{1}(z)+\alpha_{1}(z+\eta)-2 \beta_{1}(z)-\beta_{1}(z+\eta)} \\
& +a_{1}\left(a_{4}-1\right)^{2} e^{\alpha_{1}(z)-2 \beta_{1}(z)-\beta_{1}(z+\eta)}+\left(a_{3} a_{4}-a_{1}\right) e^{\alpha_{1}(z)} \\
& +\left(a_{3} a_{4}-a_{1}\right) a_{4}^{2} e^{2 \alpha_{1}(z)+\alpha_{1}(z+\eta)-\beta_{1}(z+\eta)-\beta_{1}(z)} \\
& +\left(a_{3} a_{4}-a_{1}\right) a_{4}^{2} e^{\alpha_{1}(z+\eta)-\beta_{1}(z)-\beta_{1}(z+\eta)} \\
& -2\left(a_{3} a_{4}+a_{1} a_{4}^{2}-2 a_{3} a_{4}^{2}-a_{1}\right) e^{\alpha_{1}(z)-\beta_{1}(z)}=0
\end{aligned}
$$

for all $z \in \mathbb{C}$.

If additionally $a_{3}=0$, then from (3.125) we get $a_{4}=2$, and so (3.126) can be rewritten as

$$
\begin{aligned}
& 4 e^{\alpha_{1}(z+\eta)-\beta_{1}(z+\eta)}+4 e^{-\beta_{1}(z)}-4 e^{-\beta_{1}(z+\eta)}-e^{\alpha_{1}(z)-2 \beta_{1}(z)} \\
& -4 e^{2 \alpha_{1}(z)-2 \beta_{1}(z)-\beta_{1}(z+\eta)}+4 e^{2 \alpha_{1}(z)-\beta_{1}(z)}+e^{\alpha_{1}(z)-\beta_{1}(z+\eta)} \\
& +6 e^{\alpha_{1}(z)-\beta_{1}(z)-\beta_{1}(z+\eta)}+4 e^{2 \alpha_{1}(z)+\alpha_{1}(z+\eta)-2 \beta_{1}(z)-\beta_{1}(z+\eta)} \\
& +e^{\alpha_{1}(z)-2 \beta_{1}(z)-\beta_{1}(z+\eta)}-e^{\alpha_{1}(z)}-4 e^{2 \alpha_{1}(z)+\alpha_{1}(z+\eta)-\beta_{1}(z+\eta)-\beta_{1}(z)} \\
& -4 e^{\alpha_{1}(z+\eta)-\beta_{1}(z)-\beta_{1}(z+\eta)}-6 e^{\alpha_{1}(z)-\beta_{1}(z)}=0
\end{aligned}
$$

for all $z \in \mathbb{C}$. Multiplied by $e^{-2 \alpha_{1}(z)-\alpha_{1}(z+\eta)+\beta_{1}(z)+\beta_{1}(z+\eta)}$ on two sides of (3.127), we have

$$
\begin{aligned}
& 4 e^{\beta_{1}(z)-2 \alpha_{1}(z)}+4 e^{\beta_{1}(z+\eta)-2 \alpha_{1}(z)-\alpha_{1}(z+\eta)}-4 e^{\beta_{1}(z)-2 \alpha_{1}(z)-\alpha_{1}(z+\eta)} \\
& -e^{-\alpha_{1}(z)-\alpha_{1}(z+\eta)-\beta_{1}(z)+\beta_{1}(z+\eta)}-4 e^{-\beta_{1}(z)-\alpha_{1}(z+\eta)}+4 e^{\beta_{1}(z+\eta)-\alpha_{1}(z+\eta)} \\
& +e^{\beta_{1}(z)-\alpha_{1}(z)-\alpha_{1}(z+\eta)}+6 e^{-\alpha_{1}(z)-\alpha_{1}(z+\eta)}+4 e^{-\beta_{1}(z)}+e^{-\alpha_{1}(z)-\alpha_{1}(z+\eta)-\beta_{1}(z)} \\
& -e^{\beta_{1}(z)-\alpha_{1}(z)+\beta_{1}(z+\eta)-\alpha_{1}(z+\eta)}-4 e^{-2 \alpha_{1}(z)}-6 e^{\beta_{1}(z+\eta)-\alpha_{1}(z)-\alpha_{1}(z+\eta)}=4
\end{aligned}
$$


for all $z \in \mathbb{C}$. Proceeding as in the proof of Subcase 2.2.1 and applying the above supposition we know that every term of the left side of (3.128) is not a constant. This together with Lemma 2.4 gives a contradiction. Next we suppose that $a_{3} \neq 0$. Multiplied by $e^{2 \beta_{1}(z)-2 \alpha_{1}(z)}$ on two sides of (3.126), we have

$$
\begin{aligned}
& (3.129) \\
& a_{1} a_{4}^{2} e^{\alpha_{1}(z+\eta)-2 \alpha_{1}(z)+2 \beta_{1}(z)-\beta_{1}(z+\eta)}+\left(a_{1} a_{4}^{2}+a_{3} a_{4}\right) e^{\beta_{1}(z)-2 \alpha_{1}(z)} \\
& +\left(a_{3} a_{4}-a_{1} a_{4}^{2}\right) e^{2 \beta_{1}(z)-\beta_{1}(z+\eta)-2 \alpha_{1}(z)} \\
& +2 a_{4}\left(a_{1} a_{4}-2 a_{1}+a_{3}\right) e^{\alpha_{1}(z+\eta)-\beta_{1}(z+\eta)+\beta_{1}(z)-\alpha_{1}(z)}+\left(a_{3} a_{4}-a_{1}\right) e^{-\alpha_{1}(z)} \\
& +\left(a_{3} a_{4}-a_{1} a_{4}^{2}\right) e^{-\beta_{1}(z+\eta)}+\left(2 a_{1} a_{4}-a_{3} a_{4}-a_{1} a_{4}^{2}\right) e^{\alpha_{1}(z+\eta)-\beta_{1}(z+\eta)+2 \beta_{1}(z)-\alpha_{1}(z)} \\
& +\left(a_{1} a_{4}^{2}-2 a_{3} a_{4}^{2}+a_{3} a_{4}\right) e^{\beta_{1}(z)}-a_{3} a_{4}\left(a_{4}-1\right)^{2} e^{\beta_{1}(z)-\beta_{1}(z+\eta)} \\
& +a_{1}\left(a_{4}-1\right)^{2} e^{2 \beta_{1}(z)-\alpha_{1}(z)-\beta_{1}(z+\eta)}+a_{4}\left(a_{1}-a_{3}\right)\left(a_{4}+1\right) e^{\beta_{1}(z)-\alpha_{1}(z)-\beta_{1}(z+\eta)} \\
& -a_{3} a_{4}\left(a_{4}-1\right)^{2} e^{\beta_{1}(z)-2 \alpha_{1}(z)-\beta_{1}(z+\eta)}+a_{1} a_{4}^{2} e^{\alpha_{1}(z+\eta)-\beta_{1}(z+\eta)} \\
& +a_{1}\left(a_{4}-1\right)^{2} e^{-\beta_{1}(z+\eta)-\alpha_{1}(z)}+\left(a_{3} a_{4}-a_{1}\right) e^{2 \beta_{1}(z)-\alpha_{1}(z)} \\
& +\left(a_{3} a_{4}-a_{1}\right) a_{4}^{2} e^{\alpha_{1}(z+\eta)-\beta_{1}(z+\eta)+\beta_{1}(z)} \\
& +\left(a_{3} a_{4}-a_{1}\right) a_{4}^{2} e^{\alpha_{1}(z+\eta)-\beta_{1}(z+\eta)+\beta_{1}(z)-2 \alpha_{1}(z)} \\
& -2\left(a_{3} a_{4}+a_{1} a_{4}^{2}-2 a_{3} a_{4}^{2}-a_{1}\right) e^{\beta_{1}(z)-\alpha_{1}(z)}=a_{3} a_{4} .
\end{aligned}
$$

From $(3.125)$ and $a_{3} \neq 0, a_{4} \neq 0$ we know that

$$
2 a_{\operatorname{deg}\left(\alpha_{1}\right)}=3 b_{\operatorname{deg}\left(\beta_{1}\right)},
$$

where and in what follows, $a_{\operatorname{deg}\left(\alpha_{1}\right)}$ and $b_{\operatorname{deg}\left(\beta_{1}\right)}$ denote the coefficients of the highest terms of $\alpha_{1}$ and $\beta_{1}$ respectively. From (3.130) we know that every non-vanished term of the left side of (3.129) is not a constant. This together with Lemma 2.4 gives $a_{3} a_{4}=0$, which is impossible.

If $e^{\alpha_{1}(z)+\alpha_{1}(z+\eta)-2 \beta_{1}(z)-\beta_{1}(z+\eta)}$ is not a constant, $e^{2 \alpha_{1}(z)+\alpha_{1}(z+\eta)-\beta_{1}(z)-\beta_{1}(z+\eta)}$ is a constant. In the same manner as above we can get a contradiction.

Subcase 2.2.4. Suppose that

$$
\begin{aligned}
& e^{\alpha_{1}(z)-2 \beta_{1}(z)}, e^{2 \alpha_{1}(z)-\beta_{1}(z)}, e^{\alpha_{1}(z)+\alpha_{1}(z+\eta)-\beta_{1}(z+\eta)}, e^{\alpha_{1}(z)-\beta_{1}(z+\eta)}, \\
& e^{\alpha_{1}(z)-\beta_{1}(z)-\beta_{1}(z+\eta)}, e^{\alpha_{1}(z)-2 \beta_{1}(z)-\beta_{1}(z+\eta)}, e^{\alpha_{1}(z+\eta)-\beta_{1}(z)-\beta_{1}(z+\eta)}, \\
& e^{2 \alpha_{1}(z)-2 \beta_{1}(z)-\beta_{1}(z+\eta)}, e^{2 \alpha_{1}(z)-\beta_{1}(z)-\beta_{1}(z+\eta)}, e^{\alpha_{1}(z)+\alpha_{1}(z+\eta)-2 \beta_{1}(z)-\beta_{1}(z+\eta)}, \\
& e^{2 \alpha_{1}(z)+\alpha_{1}(z+\eta)-\beta_{1}(z)-\beta_{1}(z+\eta)} \text { are not constants. Then from (3.113), Lemma }
\end{aligned}
$$

2.4 and the above supposition we get $a_{3} a_{4}=0$, and so $a_{3}=0$. Hence (3.113) can be rewritten as

$$
\begin{aligned}
& a_{4}^{2} e^{2 \beta_{1}(z)-2 \alpha_{1}(z)}+a_{4}^{2} e^{\beta_{1}(z)+\beta_{1}(z+\eta)-2 \alpha_{1}(z)-\alpha_{1}(z+\eta)}-a_{4}^{2} e^{2 \beta_{1}(z)-2 \alpha_{1}(z)-\alpha_{1}(z+\eta)} \\
& +2 a_{4}\left(a_{4}-2\right) e^{\beta_{1}(z)-\alpha_{1}(z)}-e^{\beta_{1}(z+\eta)-\alpha_{1}(z)-\alpha_{1}(z+\eta)}+\left(2 a_{4}-a_{4}^{2}\right) e^{-\alpha_{1}(z)} \\
& -a_{4}^{2} e^{-\alpha_{1}(z+\eta)}+\left(2 a_{4}-a_{4}^{2}\right) e^{2 \beta_{1}(z)-\alpha_{1}(z)}+a_{4}^{2} e^{\beta_{1}(z)+\beta_{1}(z+\eta)-\alpha_{1}(z+\eta)}
\end{aligned}
$$




$$
\begin{aligned}
& +\left(a_{4}-1\right)^{2} e^{2 \beta_{1}(z)-\alpha_{1}(z)-\alpha_{1}(z+\eta)}+a_{4}\left(a_{4}+1\right) e^{\beta_{1}(z)-\alpha_{1}(z)-\alpha_{1}(z+\eta)} \\
& +\left(a_{4}-1\right)^{2} e^{-\alpha_{1}(z)-\alpha_{1}(z+\eta)}-e^{\alpha_{1}(z)}-a_{4}^{2} e^{\beta_{1}(z)}-a_{4}^{2} e^{\beta_{1}(z)-2 \alpha_{1}(z)} \\
& -2\left(a_{4}^{2}-1\right) e^{\beta_{1}(z)-\alpha_{1}(z)+\beta_{1}(z+\eta)-\alpha_{1}(z+\eta)}=-a_{4}^{2}
\end{aligned}
$$

for all $z \in \mathbb{C}$. Next in the same manner as in Subcase 2.2.1 we can prove that every non-vanished term of the left side of (3.131) is not a constant. This together with Lemma 2.4 gives $-a_{4}^{2}=0$, and so $a_{4}=0$, which is impossible.

Acknowledgements. The authors wish to express their thanks to the referee for his/her valuable suggestions and comments.

\section{References}

[1] A. H. H. Al-khaladi, Meromorphic functions that share three values with one share value for their derivatives, J. Math. (Wuhan) 20 (2000), no. 2, 156-160.

[2] Y. M. Chiang and S. J. Feng, On the Nevanlinna characteristic of $f(z+\eta)$ and difference equations in the complex plane, Ramanujan J. 16 (2008), no. 1, 105-129.

[3] R. G. Halburd and R. J. Korhonen, Nevanlinna theory for the difference operator, Ann. Acad. Sci. Fenn. 31 (2006), no. 2, 463-478.

[4] _ Difference analogue of the lemma on the logarithmic derivative with applications to difference equations, J. Math. Anal. Appl. 314 (2006), no. 2, 477-487.

[5] W. K. Hayman, Meromorphic Functions, Clarendon Press, Oxford, 1964.

[6] J. Heittokangas, R. Korhonen, I. Laine, and J. Rieppo, Uniqueness of meromorphic functions sharing values with their shifts, Complex Var. Elliptic Equ. 56 (2011), no. $1-4,81-92$.

[7] J. Heittokangas, R. Korhonen, I. Laine, J. Rieppo, and J. L. Zhang, Value sharing results for shifts of meromorphic functions and sufficient conditions for periodicity, J. Math. Anal. Appl. 355 (2009), no. 1, 352-363.

[8] I. Lahiri, Weighted sharing of three values and uniqueness of meromorphic functions, Kodai Math. J. 24 (2001), no. 3, 421-435.

[9] I. Lahiri and A. Sarkar, On a uniqueness theorem of Tohge, Arch. Math. (Basel) 84 (2005), no. 5, 461-469.

[10] I. Laine, Nevanlinna Theory and Complex Differential Equations, Walter de Gruyter, Berlin/New York, 1993.

[11] I. Laine and C. C. Yang, Clunie theorems for difference and q-difference polynomials, J. London Math. Soc. 76 (2007), no. 3, 556-566.

[12] - Value distribution of difference polynomials, Proc. Japan Acad. Ser. A Math. Sci. 83 (2007), no. 8, 148-151.

[13] X. M. Li and Z. T. Wen, Uniqueness theorems of meromorphis functions sharing three values, Complex Var. Elliptic Equ. 56 (2011), no. 1-4, 215-232.

[14] A. Z. Mokhon'ko, On the Nevanlinna characteristics of some meromorphic functions, In: Theory of Functions, Functional Analysis and Their Applications vol. 14, 83-87, Izd-vo Khar'kovsk, Un-ta, Kharkov, 1971.

[15] J. M. Whittaker, Interpolatory Function Theory, Cambridge Tract No. 33, Cambridge University Press, 1964.

[16] C. C. Yang and H. X. Yi, Uniqueness Theory of Meromorphic Functions, Kluwer Academic Publishers, Dordrecht/Boston/London, 2003.

[17] H. X. Yi, Unicity theorems for meromorphic functions that share three values, Kodai Math. J. 18 (1995), no. 2, 300-314.

[18] - Meromorphic functions with weighted sharing of three values, Complex Var. Theory Appl. 50 (2005), no. 12, 923-934. 
[19] J. L. Zhang, Value distribution and shared sets of differences of meromorphic functions, J. Math. Anal. Appl. 367 (2010), no. 2, 401-408.

XIAO-Min Li

Department of Mathematics

OCEAN University OF China

Qingdao, Shandong 266100, P. R. China

AND

Department of Physics and Mathematics

University OF EASTERN Finland

P.O.Box 111, FI-80101 Joensud, Finland

E-mail address: lixiaomin@ouc.edu.cn

Hong-Xun Yi

Department of Mathematics

SHANDONG UNIVERSITY

Jinan, Shandong 250100, P. R. China

E-mail address: hxyi@sdu.edu.cn

CONG-Yun KANG

Department of Mathematics

Ocean University of China

Qingdao, Shandong 266100, P. R. China

E-mail address: yunyun107@163.com 